\title{
THE EFFECT ON THE KIDNEY OF BILATERAL SPLANCHNICECTOMY IN PATIENTS WITH HYPERTENSION
}

\author{
By R. H. FREYBERG AND M. M. PEET \\ (From the Departments of Internal Medicine and Surgery, Medical School, University of \\ Michigan, Ann Arbor)
}

(Received for publication August 21, 1936)

Recently there has been a renewed interest in the etiology and treatment of hypertension (hypertensive vascular disease) and its relationship to the kidneys. A surgical method of treatment of hypertension which was devised by Peet (1) consists of a bilateral resectioning of the major and minor splanchnic nerves and of the lower dorsal sympathetic chain including the 10th, 11th and 12th ganglia, supradiaphragmatically. This procedure has been employed in a sufficient number of cases so that the trend of results is indicated. It is the purpose of this communication to report the effect of this operation upon the kidneys, as it is shown by measurement of renal function and urinary abnormalities, and to correlate this effect with the blood pressure changes. Considerations of the rationale behind this treatment of hypertension, selection of candidates, technical procedure, and results other than the renal effect will not be discussed.

\section{METHOD OF STUNY}

Every candidate for this operation was carefully studied in order to exclude all cases having hypertension secondary to known organic disease. Special care was taken to exclude those patients with hemorrhagic (glomerular) nephritis having elevated blood pressure. As a result only cases considered to have primary (" arterial") hypertension (hypertensive vascular disease), were treated by splanchnicectomy. This report includes observations on only those patients whom we have studied over a period of three months, or longer, after operation.

Before, and at intervals after this operation, blood pressure and renal function were measured, and the urine was analyzed. The blood pressure was in every case measured with a mercury manometer several times while the patient was lying down. Renal function was measured by the urea clearance test of Van Slyke and Cope
(2) and by the Lashmet-Newburgh concentration test (3). Proteinuria was measured by sulphosalicylic acid precipitation (4), and the formed elements in the urinary sediment were counted by the Addis method (5).

Criteria for classification. We have adopted the normal values found by the originators of the tests of renal function, i.e., for the concentration test a nonprotein specific gravity of the urine of 1.029 or more, and for the urea clearance, values between 75 and 125 per. cent of the mean normal. The renal function was considered to be impaired when the result of either of the functional tests was below normal. A significant change in renal function was considered to consist of a change in specific gravity of 0.003 or more, or a change of 15 per cent or more in urea clearance.

It is always difficult to discuss changes in blood pressure because of the fluctuations encountered. For the purpose of classification, we have grouped the patients according to the change in blood pressure following splanchnicectomy as follows: 1, those whose blood pressure was reduced to $160 / 100 \mathrm{~mm}$. Hg, or less; 2, those whose blood pressures did not remain below $160 / 100$, but whose systolic blood pressure was reduced more than $60 \mathrm{~mm}$. $\mathrm{Hg}$, or whose diastolic pressure was reduced more than $30 \mathrm{~mm}$.; 3, those whose systolic blood pressure was reduced from 30 to 60 $\mathrm{mm}$., or whose diastolic pressure was reduced 15 to $30 \mathrm{~mm}$. $\mathrm{Hg}$.

\section{RESULTS}

By this method we have now studied fortyeight cases. The data on these cases are presented in Table I. A summary of the relationship between the renal status and the blood pressure will be found in Table II.

In almost every case there was a sharp fall in blood pressure to normal or below, immediately after splanchnicectomy. It then followed one of several courses: in some it remained approx- 
R. H. FREYBERG AND M. M. PEET

TABLE I

Data on each of the 48 cases studied, showing pre- and postoperative renal activity and blood pressure

\begin{tabular}{|c|c|c|c|c|c|c|c|c|c|c|c|}
\hline \multirow{2}{*}{$\begin{array}{c}\text { Case } \\
\text { number } \\
\text { and } \\
\text { initials }\end{array}$} & \multirow{2}{*}{ Sex } & \multirow{2}{*}{$\begin{array}{l}\text { Age at } \\
\text { time } \\
\text { of } \\
\text { oper- } \\
\text { ation }\end{array}$} & \multirow{2}{*}{$\begin{array}{c}\text { Known } \\
\text { duration } \\
\text { of } \\
\text { hyper- } \\
\text { tension }\end{array}$} & \multirow{2}{*}{$\begin{array}{c}\text { Time of } \\
\text { observation }\end{array}$} & \multirow{2}{*}{$\begin{array}{c}\text { Blood } \\
\text { pressure }\end{array}$} & \multirow{2}{*}{$\begin{array}{c}\text { Concen- } \\
\text { trating } \\
\text { ability of } \\
\text { kidneys }\end{array}$} & \multirow{2}{*}{$\begin{array}{l}\text { Urea } \\
\text { clear- } \\
\text { ance }\end{array}$} & \multicolumn{2}{|c|}{ Urine } & \multirow{2}{*}{$\begin{array}{c}\text { Classifi- } \\
\text { cation } \\
\text { in } \\
\text { Tabble } \\
\text { II }\end{array}$} & \multirow{2}{*}{ Remarks } \\
\hline & & & & & & & & $\begin{array}{c}\text { Protein- } \\
\text { uria }\end{array}$ & $\underset{\text { turia }}{\text { Hema- }}$ & & \\
\hline W. $\stackrel{1}{\mathrm{~L}}$. & $\mathbf{M}$ & \begin{tabular}{|c|} 
years \\
29
\end{tabular} & $9 \mathrm{mos}$. & \begin{tabular}{|c|} 
Pre-op. \\
2 wks. postop. \\
6 wks. postop. \\
\\
5 mos. postop. \\
11 mos. postop. \\
15 mos. postop. \\
29 mos. postop.
\end{tabular} & $\begin{array}{l}m m . \mathrm{Hg} \\
\\
236 / 166 \\
134 / 90 \\
140-160 \\
100-116 \\
138 / 90 \\
150 / 90 \\
156 / 100\end{array}$ & $\begin{array}{c}\text { nonprotein } \\
\text { specific } \\
\text { gravity } \\
1.014 \\
1.022 \\
1.026 \\
\\
1.033 \\
1.033 \\
1.031\end{array}$ & \begin{tabular}{|c|} 
per cent \\
of mean \\
normal \\
106 \\
58 \\
80 \\
\\
108 \\
125 \\
95 \\
\end{tabular} & \begin{tabular}{|c|} 
per cent \\
0.3 \\
Trace \\
0 \\
0 \\
0 \\
0
\end{tabular} & 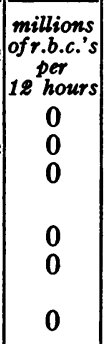 & $1 \mathrm{Ab}$ & \\
\hline M. $\stackrel{2}{\mathrm{P}}$. & F & 41 & 5 yrs. & $\begin{array}{c}\text { Pre-op. } \\
3 \text { wks. postop. } \\
3 \text { mos. postop. } \\
4 \frac{1}{2} \text { mos. postop. }\end{array}$ & $\begin{array}{l}\frac{205-240}{125-130} \\
120 / 80 \\
140-160 \\
97-100 \\
146 / 94\end{array}$ & 1.030 & $\begin{array}{l}59 \\
91 \\
79\end{array}$ & $\begin{array}{c}\text { Trace } \\
0 \\
0\end{array}$ & $\begin{array}{l}0 \\
0 \\
0\end{array}$ & $1 \mathrm{Ab}$ & \\
\hline J. J. & $\mathbf{M}$ & 45 & $1 \frac{1}{2}$ yrs. & $\begin{array}{c}\text { Pre-op. } \\
3 \text { wks. postop. } \\
6 \text { mos. postop. }\end{array}$ & $\begin{array}{l}\frac{190-230}{100-150} \\
148 / 96 \\
\frac{142-160}{98-100}\end{array}$ & 1.031 & 75 & 0 & 0 & $1 \mathrm{Ab}$ & \\
\hline V. E. & F & 22 & 3 yrs. & \begin{tabular}{|c|} 
Pre-op. \\
2 wks. postop. \\
2 mos. postop. \\
5 mos. postop. \\
8 mos. postop. \\
11 mos. postop.
\end{tabular} & $\begin{array}{l}280 / 190 \\
150 / 110 \\
146 / 102 \\
150 / 120 \\
136 / 90 \\
132 / 95\end{array}$ & $\begin{array}{l}1.033 \\
1.025 \\
1.028 \\
1.029\end{array}$ & $\begin{array}{l}87 \\
38 \\
82 \\
85\end{array}$ & $\begin{array}{l}0.12 \\
\text { Trace } \\
\text { Sl. tr. } \\
\text { Sl. tr. }\end{array}$ & $\begin{array}{l}2.2 \\
0 \\
0 \\
0\end{array}$ & $1 \mathrm{Aa}$ & \\
\hline P. $\mathbf{5}$. & $\mathbf{M}$ & 46 & 15 yrs. & $\begin{array}{c}\text { Pre-op. } \\
1 \text { mo. postop. } \\
4 \text { mos. postop. }\end{array}$ & $\begin{array}{l}\frac{180-190}{110-120} \\
140 / 90 \\
140 / 88\end{array}$ & $\begin{array}{l}1.028 \\
1.0255\end{array}$ & $\begin{array}{l}91 \\
92\end{array}$ & $\begin{array}{l}0 \\
0\end{array}$ & $\begin{array}{l}0 \\
0\end{array}$ & $1 \mathrm{Ac}$ & $\begin{array}{l}\text { Bladder residual at time } \\
\text { of concentration test }\end{array}$ \\
\hline G. $\stackrel{6}{\text { P. }}$ & F & 23 & 2 yrs. & $\begin{array}{c}\text { Pre-op. } \\
2 \text { wks. postop. } \\
4 \text { mos. postop. }\end{array}$ & $\begin{array}{l}\frac{248-270}{138} \\
180 / 120 \\
160 / 100\end{array}$ & $\begin{array}{l}1.026 \\
1.026\end{array}$ & 80 & $\begin{array}{c}\text { Trace } \\
0\end{array}$ & 0 & $1 \mathrm{Ac}$ & \\
\hline C. ${ }^{7}$. & $\mathbf{M}$ & 35 & $7 \mathrm{mos}$ & \begin{tabular}{|c|} 
Pre-op. \\
2 wks. postop. \\
$2 \frac{1}{2}$ mos. postop. \\
6 mos. postop. \\
9 mos. postop. \\
16 mos. postop.
\end{tabular} & $\begin{array}{l}268 / 176 \\
180 / 130 \\
200 / 128 \\
190 / 130 \\
180 / 125 \\
180 / 140 \\
\end{array}$ & $\begin{array}{l}1.019 \\
1.020 \\
1.030 \\
1.030 \\
1.030\end{array}$ & $\begin{array}{r}40 \\
95 \\
113 \\
85 \\
125 \\
98 \\
\end{array}$ & \begin{tabular}{|l|}
0.3 \\
0.05 \\
Trace \\
Trace \\
0 \\
0 \\
\end{tabular} & \begin{tabular}{|c|}
280 \\
0 \\
0 \\
0 \\
0 \\
0 \\
\end{tabular} & $1 \mathrm{Bb}$ & \\
\hline S. B. & $\mathbf{M}$ & 53 & 4 yrs. & \begin{tabular}{|c|} 
Pre-op. \\
3 wks. postop. \\
5 mos. postop. \\
8 mos. postop. \\
10 mos. postop. \\
12 mos. postop. \\
14 mos. postop. \\
17 mos. postop.
\end{tabular} & $\begin{array}{c}\frac{230-260}{170-180} \\
\frac{125-130}{90} \\
\frac{160-166}{109} \\
160 / 110 \\
\frac{150-165}{105-110} \\
145 / 100 \\
150 \\
\frac{105-110}{148 / 110}\end{array}$ & $\begin{array}{l}1.023 \\
1.022 \\
1.027 \\
1.026\end{array}$ & $\begin{array}{l}70 \\
51 \\
77 \\
83\end{array}$ & $\begin{array}{c}0.1 \\
\text { Trace } \\
\text { Trace } \\
\text { Trace }\end{array}$ & $\begin{array}{l}3.7 \\
0 \\
0 \\
0\end{array}$ & $1 \mathrm{Bb}$ & \\
\hline
\end{tabular}


TABLE I-Continued

\begin{tabular}{|c|c|c|c|c|c|c|c|c|c|c|c|}
\hline \multirow{2}{*}{$\begin{array}{c}\text { Case } \\
\text { number } \\
\text { and } \\
\text { initials }\end{array}$} & \multirow{2}{*}{ Sex } & \multirow{2}{*}{$\mid \begin{array}{c}\text { Age at } \\
\text { time } \\
\text { of } \\
\text { oper- } \\
\text { ation }\end{array}$} & \multirow{2}{*}{$\begin{array}{c}\text { Known } \\
\text { duration } \\
\text { of } \\
\text { hyper- } \\
\text { tension }\end{array}$} & \multirow{2}{*}{$\begin{array}{c}\text { Time of } \\
\text { observation }\end{array}$} & \multirow{2}{*}{$\begin{array}{c}\text { Blood } \\
\text { pressure }\end{array}$} & \multirow{2}{*}{$\begin{array}{c}\text { Concen- } \\
\text { trating } \\
\text { ability of } \\
\text { kidneys }\end{array}$} & \multirow{2}{*}{$\begin{array}{l}\text { Urea } \\
\text { clear- } \\
\text { ance }\end{array}$} & \multicolumn{2}{|c|}{ Urine } & \multirow{2}{*}{$\begin{array}{c}\text { Classifi- } \\
\text { cation } \\
\text { in } \\
\text { Table } \\
\text { II }\end{array}$} & \multirow{2}{*}{ Remarks } \\
\hline & & & & & & & & $\begin{array}{c}\text { Protein- } \\
\text { uria }\end{array}$ & $\underset{\text { turia }}{\text { Hema- }}$ & & \\
\hline E. $\stackrel{9}{\mathrm{G}}$. & $F$ & \begin{tabular}{|c|} 
years \\
46
\end{tabular} & 3 yrs. & $\begin{array}{c}\text { Pre-op. } \\
2 \text { wks. postop. } \\
6 \text { mos. postop. }\end{array}$ & $\begin{array}{l}m m . H g \\
250 / 134 \\
212 / 100 \\
150 / 100\end{array}$ & $\begin{array}{c}\text { nonprotein } \\
\text { specific } \\
\text { gravity } \\
1.024 \\
1.034\end{array}$ & $\begin{array}{c}\text { per cent } \\
\text { of mean } \\
\text { normal } \\
72 \\
136\end{array}$ & $\mid$\begin{tabular}{c|} 
per cent \\
0.05 \\
0
\end{tabular} & \begin{tabular}{|c|} 
millions \\
of $r . b . c$. \\
per \\
12 hours \\
0 \\
1.25
\end{tabular} & $1 \mathrm{Bb}$ & \\
\hline M. W. & $\mathrm{F}$ & 44 & 3 yrs. & $\begin{array}{c}\text { Pre-op. } \\
2 \text { wks. postop. } \\
3 \text { mos. postop. } \\
6 \text { mos. postop. }\end{array}$ & $\begin{array}{l}\frac{250-260}{150-162} \\
140 / 95 \\
\frac{178-220}{110-120} \\
\frac{186-200}{112}\end{array}$ & $\begin{array}{l}1.023 \\
1.027 \\
1.028\end{array}$ & $\begin{array}{r}77 \\
86 \\
111\end{array}$ & $\begin{array}{c}\text { Trace } \\
0 \\
0\end{array}$ & $\begin{array}{l}0 \\
0\end{array}$ & $1 \mathrm{Bb}$ & \\
\hline $\begin{array}{c}11 \\
\text { E. } \mathrm{H} .\end{array}$ & $\mathrm{F}$ & 23 & 6 yrs. & \begin{tabular}{|c|} 
Pre-op. \\
2 wks. postop. \\
2 mos. postop. \\
6 mos. postop.
\end{tabular} & $\begin{array}{l}230-240 \\
140-160 \\
180 / 130 \\
176 / 128 \\
170 / 128\end{array}$ & $\begin{array}{l}1.026 \\
1.030 \\
1.033\end{array}$ & $\begin{array}{l}84 \\
88 \\
89\end{array}$ & $\begin{array}{l}\text { Trace } \\
\text { Trace } \\
\text { Trace }\end{array}$ & $\begin{array}{l}0 \\
0 \\
0\end{array}$ & $1 \mathrm{Bb}$ & \\
\hline $\begin{array}{c}12 \\
\text { O. Mc. }\end{array}$ & $\mathbf{M}$ & 44 & $6 \mathrm{mos}$ & $\begin{array}{c}\text { Pre-op. } \\
2 \text { wks. postop. } \\
2 \text { mos. postop. } \\
3 \text { mos. postop. } \\
6 \text { mos. postop. }\end{array}$ & $\begin{array}{l}\frac{224-256}{140-172} \\
\frac{170}{110-116} \\
150 / 110 \\
170 / 116 \\
190 / 124\end{array}$ & $\begin{array}{l}1.028 \\
1.024\end{array}$ & $\begin{array}{r}84 \\
97 \\
120\end{array}$ & $\begin{array}{l}\text { Trace } \\
\text { Trace } \\
\text { Sl. tr. }\end{array}$ & $\begin{array}{c}0 \\
1.3\end{array}$ & $1 \mathrm{Bb}$ & \\
\hline $\begin{array}{r}13 \\
\text { A. } B .\end{array}$ & F & 40 & 6 yrs. & $\begin{array}{c}\text { Pre-op. } \\
1 \text { mo. postop. } \\
6 \text { mos. postop. }\end{array}$ & $\begin{array}{l}\frac{244-276}{148-156} \\
160 / 120 \\
178 / 112\end{array}$ & 1.036 & $\begin{array}{r}97 \\
103\end{array}$ & $\begin{array}{c}\text { Trace } \\
0\end{array}$ & 0 & $1 \mathrm{Ba}$ & \\
\hline $\begin{array}{r}14 \\
\text { H. R. }\end{array}$ & F & 49 & 2 yrs. & $\begin{array}{c}\text { Pre-op. } \\
3 \text { wks. postop. } \\
5 \text { mos. postop. } \\
9 \text { mos. postop. }\end{array}$ & $\begin{array}{l}\frac{210-252}{120-148} \\
160 / 100 \\
155 / 100 \\
174 / 105\end{array}$ & $\begin{array}{l}1.030 \\
\\
1.032 \\
1.029\end{array}$ & 101 & $\begin{array}{c}\text { Trace } \\
0 \\
0\end{array}$ & $\begin{array}{l}0 \\
0\end{array}$ & $1 \mathrm{Ba}$ & \\
\hline F. $\stackrel{15}{M .}$ & $\mathbf{M}$ & 38 & $1 \mathrm{yr}$. & \begin{tabular}{|c|} 
Pre-op. \\
2 wks. postop. \\
5 mos. postop.
\end{tabular} & $\begin{array}{l}\frac{240-260}{145-150} \\
210 / 115 \\
\frac{210}{120-130}\end{array}$ & $\begin{array}{l}1.025 \\
1.027 \\
1.029\end{array}$ & $\begin{array}{l}71 \\
72 \\
86\end{array}$ & $\begin{array}{c}0.4 \\
0.1 \\
\text { Trace }\end{array}$ & $\begin{array}{l}0 \\
0 \\
0\end{array}$ & $1 \mathrm{Cb}$ & \\
\hline L. $\stackrel{16}{M}$. & $\mathbf{M}$ & 31 & 4 yrs. & \begin{tabular}{|c|} 
Pre-op. \\
2 wks. postop. \\
2 mos. postop. \\
6 mos. postop. \\
16 mos. postop.
\end{tabular} & $\begin{array}{l}\frac{220-226}{145-158} \\
\frac{140-150}{90-100} \\
190 / 140 \\
\frac{180-200}{134-140} \\
\end{array}$ & $\begin{array}{l}1.024 \\
1.026 \\
1.026\end{array}$ & $\begin{array}{l}72 \\
60 \\
92\end{array}$ & $\begin{array}{l}\text { Trace } \\
\text { Trace } \\
\text { Trace }\end{array}$ & $\begin{array}{l}0 \\
0\end{array}$ & $1 \mathrm{Cb}$ & Acute coryza \\
\hline $\begin{array}{l}17 \\
\text { S. } 0 .\end{array}$ & $F$ & 33 & $?$ & $\begin{array}{c}\text { Pre-op. } \\
3 \text { wks. postop. } \\
6 \text { mos. postop. }\end{array}$ & $\begin{array}{c}\frac{210}{130-140} \\
\frac{130-160}{70-110} \\
\frac{170-198}{110-126} \\
\end{array}$ & $\begin{array}{l}1.026 \\
1.029\end{array}$ & 59 & $\begin{array}{l}\text { Trace } \\
\text { Trace }\end{array}$ & 0 & $1 \mathrm{Cb}$ & \\
\hline
\end{tabular}


TABLE I-Continued

\begin{tabular}{|c|c|c|c|c|c|c|c|c|c|c|c|}
\hline \multirow{2}{*}{$\begin{array}{c}\text { Case } \\
\text { number } \\
\text { and } \\
\text { initials }\end{array}$} & \multirow{2}{*}{ Sex } & \multirow{2}{*}{$\begin{array}{l}\text { Age at } \\
\text { time } \\
\text { of } \\
\text { oper- } \\
\text { ation }\end{array}$} & \multirow{2}{*}{$\begin{array}{c}\text { Known } \\
\text { duration } \\
\text { of } \\
\text { hyper- } \\
\text { tension }\end{array}$} & \multirow{2}{*}{$\begin{array}{c}\text { Time of } \\
\text { observation }\end{array}$} & \multirow{2}{*}{$\begin{array}{c}\text { Blood } \\
\text { pressure }\end{array}$} & \multirow{2}{*}{$\begin{array}{c}\text { Concen- } \\
\text { trating } \\
\text { ability of } \\
\text { kidneys }\end{array}$} & \multirow{2}{*}{$\begin{array}{l}\text { Urea } \\
\text { clear- } \\
\text { ance }\end{array}$} & \multicolumn{2}{|c|}{ Urine } & \multirow{2}{*}{$\begin{array}{c}\text { Classifi- } \\
\text { cation } \\
\text { in } \\
\text { Table } \\
\text { II }\end{array}$} & \multirow{2}{*}{ Remarks } \\
\hline & & & & & & & & $\begin{array}{c}\text { Protein- } \\
\text { uria }\end{array}$ & $\underset{\text { turia }}{\text { Hema- }}$ & & \\
\hline $\begin{array}{r}18 \\
\text { M. S. }\end{array}$ & $F$ & $\begin{array}{c}\text { years } \\
33\end{array}$ & 2 yrs. & $\begin{array}{c}\text { Pre-op. } \\
3 \text { wks. postop. } \\
6 \text { mos. postop. }\end{array}$ & $\begin{array}{l}m m . H g \\
190-220 \\
\frac{94-130}{90} \\
154 / 116 \\
\frac{140-220}{84-108}\end{array}$ & $\begin{array}{c}\text { nonprotein } \\
\text { specific } \\
\text { gravity } \\
1.028 \\
\\
1.033\end{array}$ & $\begin{array}{c}\text { per cent } \\
\text { of mean } \\
\text { normal } \\
76 \\
\\
92\end{array}$ & $\begin{array}{l}\text { per cent } \\
\text { Trace } \\
\text { Trace } \\
0\end{array}$ & $\mid \begin{array}{c}\text { millions } \\
\text { of r.b.c.'s. } \\
\text { per } \\
12 \text { hours } \\
0 \\
\\
0\end{array}$ & $1 \mathrm{Cb}$ & \\
\hline $\begin{array}{l}19 \\
\text { N. M. }\end{array}$ & F & 39 & 3 yrs. & \begin{tabular}{|c|} 
Pre-op. \\
2 wks. postop. \\
4 mos. postop.
\end{tabular} & $\begin{array}{l}\frac{198-260}{138} \\
\frac{200 / 130}{180-220} \\
\frac{130}{130}\end{array}$ & $\begin{array}{l}1.016 \\
1.023\end{array}$ & $\begin{array}{r}56 \\
109\end{array}$ & $\begin{array}{c}2.0 \\
\text { Trace }\end{array}$ & $\begin{array}{c}\text { Gross } \\
0\end{array}$ & $1 \mathrm{Cb}$ & \\
\hline $\begin{array}{r}20 \\
\text { H. F. }\end{array}$ & $\mathbf{M}$ & 46 & 2 yrs. & \begin{tabular}{|c|} 
Pre-op. \\
3 wks. postop. \\
8 mos. postop.
\end{tabular} & $\begin{array}{c}\frac{235-280}{126-170} \\
195 / 130 \\
\frac{220-240}{149} \\
\end{array}$ & $\begin{array}{l}1.029 \\
1.029\end{array}$ & $\begin{array}{l}89 \\
78\end{array}$ & \begin{tabular}{|l|} 
Trace \\
Trace
\end{tabular} & $\begin{array}{l}0 \\
0\end{array}$ & $1 \mathrm{Ca}$ & \\
\hline D. $\stackrel{21}{\mathrm{P}}$. & $\mathbf{M}$ & 41 & 7 yrs. & \begin{tabular}{|c|} 
Pre-op. \\
1 mo. postop. \\
10 mos. postop.
\end{tabular} & $\begin{array}{l}\frac{234-250}{150-160} \\
210 / 105 \\
210 / 136\end{array}$ & $\begin{array}{l}1.025 \\
1.024\end{array}$ & $\begin{array}{r}88 \\
100\end{array}$ & $\begin{array}{c}\text { Trace } \\
0\end{array}$ & 0 & $1 \mathrm{Cc}$ & \\
\hline E. $\stackrel{22}{\mathrm{C} .}$ & $F$ & 45 & $1 \frac{1}{2} \mathrm{yrs}$ & $\begin{array}{c}\text { Pre-op. } \\
2 \text { wks. postop. } \\
3 \text { mos. postop. } \\
6 \text { mos. postop. }\end{array}$ & $\begin{array}{l}\frac{198-244}{114-140} \\
132 / 82 \\
\frac{168 / 230}{100-130} \\
180 / 110\end{array}$ & $\begin{array}{l}1.026 \\
1.027 \\
1.028\end{array}$ & $\begin{array}{r}96 \\
108 \\
122\end{array}$ & $\begin{array}{c}0.1 \\
0 \\
\text { Sl. tr. }\end{array}$ & & $1 \mathrm{Cc}$ & \\
\hline L. $\stackrel{23}{\mathrm{~V} .}$ & $F$ & 33 & 4 yrs. & \begin{tabular}{|c|}
4 yrs. pre-op. \\
3 yrs. pre-op. \\
2 yrs. pre-op. \\
1 wk. pre-op. \\
3 wks. postop. \\
10 mos. postop.
\end{tabular} & $\begin{array}{l}158 / 100 \\
158 / 112 \\
182 / 130 \\
202 / 132 \\
130 / 90 \\
160 \\
112-120\end{array}$ & $\begin{array}{l}1.018 \\
1.016 \\
1.012 \\
1.012\end{array}$ & $\begin{array}{r}129 \\
92\end{array}$ & $\begin{array}{l}0 \\
\text { Trace } \\
\text { Trace } \\
\text { Trace } \\
\text { Trace }\end{array}$ & $\begin{array}{l}0 \\
0\end{array}$ & $1 \mathrm{Cc}$ & $\begin{array}{c}? \text { pituitary disease with } \\
\text { diabetes insipidus } \\
\end{array}$ \\
\hline L. B. & $\mathbf{M}$ & 45 & 5 yrs. & \begin{tabular}{|c|} 
Pre-op. \\
3 wks. postop. \\
14 mos. postop.
\end{tabular} & $\begin{array}{l}\frac{210-220}{120} \\
150 / 95 \\
\frac{180-190}{110-130}\end{array}$ & $\begin{array}{l}1.030 \\
1.025\end{array}$ & 71 & $\begin{array}{l}\text { Sl. tr. } \\
\text { Trace }\end{array}$ & & $1 \mathrm{Cd}$ & \\
\hline $\begin{array}{l}25 \\
\text { H. H. }\end{array}$ & $\mathbf{F}$ & 44 & 3 yrs. & $\begin{array}{c}\text { Pre-op. } \\
3 \text { wks. postop. } \\
4 \text { mos. postop. } \\
6 \text { mos. postop. } \\
21 \text { mos. postop. }\end{array}$ & $\begin{array}{c}\frac{240-248}{154-165} \\
\frac{200-240}{140} \\
235 / 130 \\
\frac{226-250}{132-142} \\
242 / 148\end{array}$ & $\begin{array}{l}1.033 \\
\\
1.029 \\
1.032 \\
1.034\end{array}$ & $\begin{array}{l}76 \\
80\end{array}$ & $\begin{array}{l}\text { Trace } \\
\text { Trace } \\
\text { Trace } \\
\text { Trace }\end{array}$ & $\begin{array}{l}\mathbf{0} \\
\mathbf{0} \\
\mathbf{0}\end{array}$ & $2 a$ & \\
\hline $\begin{array}{r}26 \\
\text { H. K. }\end{array}$ & $\mathbf{M}$ & 37 & 3 yrs. & $\begin{array}{c}\text { Pre-op. } \\
3 \text { wks. postop. } \\
8 \text { mos. postop. }\end{array}$ & $\begin{array}{l}190-226 \\
130-160 \\
180 / 110 \\
230 / 150\end{array}$ & & $\begin{array}{l}80 \\
84\end{array}$ & $\begin{array}{l}\text { Trace } \\
\text { Trace }\end{array}$ & & $2 a$ & \\
\hline
\end{tabular}


TABLE I-Continued

\begin{tabular}{|c|c|c|c|c|c|c|c|c|c|c|c|}
\hline \multirow{2}{*}{$\begin{array}{c}\text { Case } \\
\text { number } \\
\text { and } \\
\text { initials }\end{array}$} & \multirow{2}{*}{ Sex } & \multirow{2}{*}{$\begin{array}{c}\text { Age at } \\
\text { time } \\
\text { of } \\
\text { oper- } \\
\text { ation }\end{array}$} & \multirow{2}{*}{$\begin{array}{c}\text { Known } \\
\text { duration } \\
\text { of } \\
\text { hyper- } \\
\text { tension }\end{array}$} & \multirow{2}{*}{$\begin{array}{l}\text { Time of } \\
\text { observation }\end{array}$} & \multirow{2}{*}{$\begin{array}{l}\text { Blood } \\
\text { pressure }\end{array}$} & \multirow{2}{*}{$\begin{array}{l}\text { Concen- } \\
\text { trating } \\
\text { ability of } \\
\text { kidneys }\end{array}$} & \multirow{2}{*}{$\begin{array}{l}\text { Urea } \\
\text { clear- } \\
\text { ance }\end{array}$} & \multicolumn{2}{|c|}{ Urine } & \multirow{2}{*}{$\begin{array}{c}\text { Classifi- } \\
\text { cation } \\
\text { in } \\
T \text { able } \\
\text { II }\end{array}$} & \multirow{2}{*}{ Remark } \\
\hline & & & & & & & & $\begin{array}{c}\text { Protein- } \\
\text { uria }\end{array}$ & $\underset{\text { turia }}{\text { Hema- }}$ & & \\
\hline $\begin{array}{r}27 \\
\text { H. S. }\end{array}$ & $\mathbf{M}$ & $\begin{array}{c}\text { years } \\
36\end{array}$ & $8 \mathrm{mos}$. & \begin{tabular}{|c|} 
Pre-op. \\
3 mos. postop. \\
4 mos. postop. \\
6 mos. postop. \\
13 mos. postop. \\
24 mos. postop.
\end{tabular} & $\begin{array}{c}m m . H g \\
\frac{198-215}{120-148} \\
\frac{168-180}{120} \\
186 / 110 \\
204 / 132 \\
200 / 128 \\
\frac{194-204}{120-126}\end{array}$ & $\begin{array}{c}\text { nonprotein } \\
\text { specific } \\
\text { gravity } \\
1.018 \\
1.025 \\
\\
1.024\end{array}$ & $\begin{array}{c}\text { per cent } \\
\text { of mean } \\
\text { normal } \\
91 \\
66 \\
74\end{array}$ & $\begin{array}{c}\text { per cent } \\
0 \\
0.05 \\
\text { Trace } \\
\text { Trace }\end{array}$ & $\begin{array}{c}\text { millions } \\
\text { of r.b.c.'s } \\
\text { per } \\
12 \text { hours } \\
0 \\
0 \\
0 \\
0\end{array}$ & $2 d$ & \\
\hline $\begin{array}{r}29 \\
\text { F. N. }\end{array}$ & $F$ & 39 & 3 yrs. & \begin{tabular}{|c|} 
Pre-op. \\
3 wks. postop. \\
3 mos. postop. \\
6 mos. postop. \\
12 mos. postop. \\
21 mos. postop.
\end{tabular} & $\begin{array}{l}\frac{220-250}{130-150} \\
192 / 110 \\
220 / 130 \\
240 / 140 \\
244 / 140 \\
\frac{230-250}{152}\end{array}$ & $\begin{array}{l}1.022 \\
\\
1.023 \\
1.021 \\
1.020 \\
1.021\end{array}$ & $\begin{array}{l}35 \\
39 \\
39\end{array}$ & \begin{tabular}{|c|} 
Trace \\
\\
Trace \\
Trace \\
0.1 \\
Trace
\end{tabular} & $\begin{array}{l}0 \\
0 \\
0 \\
0\end{array}$ & $2 b$ & \\
\hline $\begin{array}{c}30 \\
\text { W. Mc. }\end{array}$ & $\mathbf{M}$ & 55 & 4 yrs. & \begin{tabular}{|c|} 
Pre-op. \\
5 wks. postop. \\
5 mos. postop. \\
12 mos. postop.
\end{tabular} & $\begin{array}{l}\frac{188-226}{120-135} \\
\frac{170-198}{122-128} \\
195 / 120 \\
\frac{185-220}{120-140}\end{array}$ & $\begin{array}{l}1.025 \\
1.029 \\
1.027 \\
1.025\end{array}$ & $\begin{array}{l}67 \\
75\end{array}$ & $\begin{array}{l}\text { Trace } \\
\text { Trace } \\
\text { Trace } \\
\text { Trace }\end{array}$ & & $2 b$ & \\
\hline $\begin{array}{r}32 \\
\text { H. B. }\end{array}$ & F & 43 & 8 yrs. & $\begin{array}{c}\text { Pre-op. } \\
2 \text { wks. postop. } \\
3 \text { mos. postop. } \\
7 \text { mos. postop. }\end{array}$ & $\begin{array}{l}\frac{200-245}{130-145} \\
220 / 120 \\
180 / 100 \\
\frac{240-260}{150}\end{array}$ & $\begin{array}{l}1.025 \\
1.024 \\
1.023\end{array}$ & $\begin{array}{l}54 \\
60\end{array}$ & \begin{tabular}{|c|}
0.1 \\
\\
Trace \\
Sl. tr.
\end{tabular} & $\begin{array}{c}3.0 \\
1.4 \\
0\end{array}$ & $2 b$ & \\
\hline M. S. & $\mathbf{F}$ & 42 & 5 yrs. & \begin{tabular}{|c|} 
Pre-op. \\
3 wks. postop. \\
10 mos. postop.
\end{tabular} & $\begin{array}{l}\frac{210-222}{130-134} \\
190 / 112 \\
\frac{180-210}{130-140}\end{array}$ & $\begin{array}{l}1.027 \\
1.026\end{array}$ & $\begin{array}{r}92 \\
102\end{array}$ & $\begin{array}{l}\text { Trace } \\
\text { Trace }\end{array}$ & & $2 \mathrm{~b}$ & \\
\hline R. $\begin{array}{l}34 \\
\text { H. }\end{array}$ & F & 44 & 4 yrs. & \begin{tabular}{|c|} 
Pre-op. \\
\\
3 wks. postop. \\
14 mos. postop.
\end{tabular} & $\begin{array}{l}208-250 \\
126-136 \\
200 / 120 \\
240 / 144\end{array}$ & 1.021 & 112 & $\begin{array}{l}\text { Trace } \\
\text { Trace }\end{array}$ & & $2 b$ & \\
\hline
\end{tabular}


TABLE I-Continued

\begin{tabular}{|c|c|c|c|c|c|c|c|c|c|c|c|}
\hline \multirow{2}{*}{$\begin{array}{c}\text { Case } \\
\text { number } \\
\text { and } \\
\text { initials }\end{array}$} & \multirow{2}{*}{ Sex } & \multirow{2}{*}{$\begin{array}{c}\text { Age at } \\
\text { time } \\
\text { of } \\
\text { oper- } \\
\text { ation }\end{array}$} & \multirow{2}{*}{$\begin{array}{c}\text { Known } \\
\text { duration } \\
\text { of } \\
\text { hyper- } \\
\text { tension }\end{array}$} & \multirow{2}{*}{$\begin{array}{c}\text { Time of } \\
\text { observation }\end{array}$} & \multirow{2}{*}{$\begin{array}{l}\text { Blood } \\
\text { pressure }\end{array}$} & \multirow{2}{*}{$\begin{array}{l}\text { Concen- } \\
\text { trating } \\
\text { ability of } \\
\text { kidneys }\end{array}$} & \multirow{2}{*}{$\begin{array}{c}\text { Urea } \\
\text { clear- } \\
\text { ance }\end{array}$} & \multicolumn{2}{|c|}{ Urine } & \multirow{2}{*}{$\begin{array}{c}\text { Classifi- } \\
\text { cation } \\
\text { in } \\
\text { Table } \\
\text { II }\end{array}$} & \multirow{2}{*}{ Remarks } \\
\hline & & & & & & & & $\begin{array}{c}\text { Protein- } \\
\text { uria }\end{array}$ & $\begin{array}{c}\text { Hema- } \\
\text { turia }\end{array}$ & & \\
\hline A. $\stackrel{35}{V}$ & $F$ & $\begin{array}{c}\text { years } \\
48\end{array}$ & $5 \mathrm{yrs}$ & $\begin{array}{c}\text { Pre-op. } \\
1 \text { mo. postop. } \\
3 \text { mos. postop. } \\
6 \text { mos. postop. }\end{array}$ & $\begin{array}{c}m m . H g \\
\frac{242-300}{160-170} \\
\frac{180-210}{120} \\
\frac{230-240}{120-130} \\
\frac{240-300}{145-160}\end{array}$ & 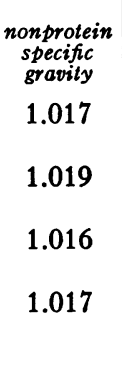 & $\begin{array}{c}\text { per cent } \\
\text { of mean } \\
\text { normal } \\
21 \\
26 \\
24 \\
19\end{array}$ & \begin{tabular}{|c|} 
per cent \\
0.25 \\
Trace \\
Trace \\
Trace
\end{tabular} & $\begin{array}{l}\text { millions } \\
\text { of r.b.c.'s } \\
\text { per. } \\
12 \text { hours } \\
\\
\\
\\
\end{array}$ & $2 b$ & \\
\hline $\begin{array}{l}36 \\
\text { I. B. }\end{array}$ & $F$ & 43 & $12 \mathrm{yrs}$. & $\begin{array}{c}\text { Pre-op. } \\
1 \text { mo. postop. } \\
1 \frac{1}{2} \text { mos. postop. } \\
6 \text { mos. postop. }\end{array}$ & $\begin{array}{l}260 / 150 \\
190 / 110 \\
240 / 160 \\
260 / 160\end{array}$ & $\begin{array}{l}1.022 \\
1.023\end{array}$ & $\begin{array}{l}45 \\
57\end{array}$ & $\begin{array}{l}0.6 \\
0.4\end{array}$ & & $2 b$ & \\
\hline F. R. $\begin{array}{r}37 \\
\text {. }\end{array}$ & $\mathbf{M}$ & 38 & 2 yrs. & \begin{tabular}{|c|} 
Pre-op. \\
2 mos. postop. \\
5 mos. postop.
\end{tabular} & $\begin{array}{l}\frac{160-178}{108} \\
170 / 110 \\
170 / 110\end{array}$ & $\begin{array}{l}1.013 \\
1.014 \\
1.015\end{array}$ & $\begin{array}{l}34 \\
27 \\
27\end{array}$ & $\begin{array}{l}0.25 \\
0.2 \\
0.22\end{array}$ & $\begin{array}{l}12 \\
16\end{array}$ & $2 b$ & $\begin{array}{l}\text { Blood nonprotein nitro- } \\
\text { gen } 56 \mathrm{mgm} \text {. per cent } \\
\text { Blood nonprotein nitro- } \\
\text { gen } 54 \mathrm{mgm} \text {. per cent }\end{array}$ \\
\hline L. $\begin{array}{l}38 \\
\text { H. }\end{array}$ & $\mathbf{M}$ & 46 & 3 yrs. & $\begin{array}{c}\text { Pre-op. } \\
3 \text { wks. postop. } \\
6 \text { mos. postop. }\end{array}$ & $\begin{array}{l}\frac{195-215}{116-130} \\
140 / 90 \\
200 / 110\end{array}$ & $\begin{array}{l}1.028 \\
1.027\end{array}$ & $\begin{array}{r}96 \\
80 \\
127\end{array}$ & $\begin{array}{l}\text { Trace } \\
\text { Trace }\end{array}$ & $\begin{array}{c}1.1 \\
0\end{array}$ & $2 b$ & \\
\hline $\begin{array}{r}39 \\
\text { W. B. }\end{array}$ & F & 43 & 2 yrs. & $\begin{array}{c}\text { Pre-op. } \\
3 \text { wks. postop. } \\
6 \text { mos. postop. } \\
9 \text { mos. postop. }\end{array}$ & $\begin{array}{l}\frac{192-240}{112-150} \\
200 / 110 \\
236 / 140 \\
210-220 \\
126-140\end{array}$ & $\begin{array}{l}1.018 \\
1.022 \\
1.019\end{array}$ & $\begin{array}{l}62 \\
71 \\
73\end{array}$ & & & $2 b$ & Slight edema present \\
\hline c. $\stackrel{40}{\text { V. }}$ & $\mathbf{M}$ & 34 & $1 \mathrm{yr}$. & \begin{tabular}{|}
3 mos. pre-op. \\
1 wk. postop. \\
3 wks. postop. \\
10 mos. postop.
\end{tabular} & $\begin{array}{l}230 / 150 \\
240 / 156 \\
180 / 130 \\
220-250 \\
150-170\end{array}$ & $\begin{array}{l}1.019 \\
1.018 \\
1.011\end{array}$ & $\begin{array}{l}47 \\
47 \\
16\end{array}$ & \begin{tabular}{l|}
0.33 \\
0.33 \\
0.3
\end{tabular} & $\begin{array}{c}0 \\
1.5 \\
0\end{array}$ & $2 c$ & \\
\hline $\begin{array}{l}41 \\
\text { I. J. }\end{array}$ & $F$ & 55 & 5 yrs. & $\begin{array}{c}\text { Pre-op. } \\
3 \text { wks. postop. } \\
10 \text { mos. postop. }\end{array}$ & $\begin{array}{l}\frac{268-300}{130-170} \\
\frac{220-270}{120} \\
\frac{270}{130-170}\end{array}$ & $\begin{array}{l}1.019 \\
1.021\end{array}$ & 38 & $\begin{array}{l}\text { Trace } \\
\text { Trace }\end{array}$ & & $2 c$ & \\
\hline E. $\begin{array}{l}42 \\
\text { H. }\end{array}$ & $F$ & 52 & $1 \mathrm{yr}$. & $\begin{array}{c}\text { Pre-op. } \\
3 \text { wks. postop. } \\
15 \text { mos. postop. }\end{array}$ & $\begin{array}{l}230 / 130 \\
160 / 110 \\
210-230 \\
120-150\end{array}$ & 1.024 & $\begin{array}{l}69 \\
49\end{array}$ & $\begin{array}{l}\text { Trace } \\
\text { Trace }\end{array}$ & & $2 c$ & \\
\hline c. $\stackrel{43}{W}$. & $\mathbf{M}$ & 45 & $6 \mathrm{mos}$ & $\begin{array}{c}\text { Pre-op. } \\
3 \text { wks. postop. } \\
10 \text { mos. postop. }\end{array}$ & $\begin{array}{l}210 / 120 \\
140 / 90 \\
185-190 \\
125-130\end{array}$ & $\begin{array}{l}1.027 \\
1.022\end{array}$ & $\begin{array}{l}91 \\
45\end{array}$ & $\begin{array}{l}\text { Sl. tr. } \\
\text { Trace }\end{array}$ & $\begin{array}{c}0 \\
1.8\end{array}$ & $2 c$ & \\
\hline
\end{tabular}


TABLE I-Continued

\begin{tabular}{|c|c|c|c|c|c|c|c|c|c|c|c|}
\hline \multirow{2}{*}{$\begin{array}{c}\text { Case } \\
\text { number } \\
\text { and } \\
\text { initials }\end{array}$} & \multirow{2}{*}{ Sex } & \multirow{2}{*}{$\begin{array}{c}\text { Age at } \\
\text { time } \\
\text { of } \\
\text { oper- } \\
\text { ation }\end{array}$} & \multirow{2}{*}{$\begin{array}{c}\text { Known } \\
\text { duration } \\
\text { of } \\
\text { hyper- } \\
\text { tension }\end{array}$} & \multirow{2}{*}{$\begin{array}{c}\text { Time of } \\
\text { observation }\end{array}$} & \multirow{2}{*}{$\underset{\text { pressure }}{\text { Blood }}$} & \multirow{2}{*}{$\begin{array}{c}\text { Concen- } \\
\text { trating } \\
\text { ability of } \\
\text { kidneys }\end{array}$} & \multirow{2}{*}{$\begin{array}{l}\text { Urea } \\
\text { clear- } \\
\text { ance }\end{array}$} & \multicolumn{2}{|c|}{ Urine } & \multirow{2}{*}{$-\begin{array}{c}\text { Classifi- } \\
\text { cation } \\
\text { in } \\
\text { Table } \\
\text { II }\end{array}$} & \multirow{2}{*}{ Remarks } \\
\hline & & & & & & & & $\begin{array}{c}\text { Protein- } \\
\text { uria }\end{array}$ & $\underset{\text { turia }}{\text { Hema- }}$ & & \\
\hline $\begin{array}{r}44 \\
\text { F. S. }\end{array}$ & $\mathbf{M}$ & $\begin{array}{c}\text { years } \\
40\end{array}$ & 17 yrs. & $\begin{array}{c}\text { Pre-op. } \\
3 \text { wks. postop. } \\
3 \text { mos. postop. } \\
7 \text { mos. postop. }\end{array}$ & $\begin{array}{c}m m . \mathrm{Hg} \\
\frac{172-234}{108-130} \\
\frac{115-130}{80-90} \\
194 / 124 \\
172 / 118\end{array}$ & $\begin{array}{c}\text { nomprotein } \\
\text { specific } \\
\text { gravity } \\
1.029\end{array}$ & $\begin{array}{c}\text { per cent } \\
\text { of mean } \\
\text { normal } \\
84 \\
91 \\
\\
82 \\
87\end{array}$ & $\begin{array}{c}\text { per cent } \\
\text { Trace } \\
0 \\
0 \\
\text { Trace }\end{array}$ & 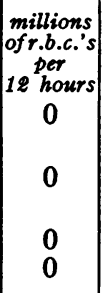 & $2 c$ & \\
\hline $\begin{array}{c}45 \\
\text { H. Mc. }\end{array}$ & F & 38 & 2 yrs. & $\begin{array}{c}\text { Pre-op. } \\
1 \text { wk. postop. } \\
5 \text { mos. postop. }\end{array}$ & $\begin{array}{l}180 / 120 \\
200 / 120 \\
205 / 130\end{array}$ & $\begin{array}{l}1.028 \\
1.021\end{array}$ & $\begin{array}{r}114 \\
89\end{array}$ & $\begin{array}{c}0.1 \\
\text { Trace }\end{array}$ & $\begin{array}{l}0 \\
0\end{array}$ & $2 c$ & \\
\hline $\begin{array}{r}46 \\
\text { F. G. }\end{array}$ & $\mathbf{M}$ & 35 & $3 \mathrm{mos}$ & $\begin{array}{c}\text { Pre-op. } \\
4 \text { wks. postop. } \\
6 \text { wks. postop. } \\
8 \text { wks. postop. }\end{array}$ & $\begin{array}{l}\frac{226-240}{150-160} \\
160 / 120 \\
\frac{250}{144-150}\end{array}$ & 1.013 & $\begin{array}{l}17 \\
13\end{array}$ & 0.3 & .9 & $2 c$ & $\begin{array}{l}\text { Nonprotein nitrogen } 58 \\
\text { mgm. per cent } \\
\\
\text { Died in uremia. Blood } \\
\text { nonprotein nitrogen } \\
114 \text { mgm. per cent }\end{array}$ \\
\hline $\begin{array}{r}47 \\
\text { L. B. }\end{array}$ & $F$ & 21 & $3 \mathrm{mos}$ & $\begin{array}{c}\text { Pre-op. } \\
2 \text { wks. postop. } \\
6 \text { wks. postop. } \\
8 \text { mos. postop. }\end{array}$ & $\begin{array}{l}220 / 146 \\
166 / 122 \\
220 / 140\end{array}$ & $\begin{array}{l}1.017 \\
1.018\end{array}$ & $\begin{array}{l}40 \\
46\end{array}$ & $\begin{array}{l}1.0 \\
0.6\end{array}$ & 2.4 & 2c & Died in uremia \\
\hline $\begin{array}{l}48 \\
\text { I. B. }\end{array}$ & $F$ & 45 & 3 yrs. & $\begin{array}{c}\text { Pre-op. } \\
6 \text { mos. postop. }\end{array}$ & $\frac{\frac{155-182}{110}}{210 / 130}$ & $\begin{array}{l}1.037 \\
1.034\end{array}$ & $\begin{array}{r}95 \\
103\end{array}$ & Sl. tr. & $\begin{array}{l}0 \\
0\end{array}$ & 3 & \\
\hline
\end{tabular}

imately normal; in others it rose to various levels even to the preoperative value. Following this secondary rise the blood pressure in some cases remained unchanged, in others it again decreased, sometimes rapidly sometimes over a period of months.

The effect of splanchnicectomy on the kidneys can best be considered in relation to the effect on the blood pressure. According to this relationship, the patients fall into different groups. Figures 1 to 8 illustrate the course of events in representative cases.

Explanation of figures. In each case the vertical line, topped by an arrow, represents the bilateral splanchnicectomy. The time charted to the left of this line refers to the known duration of hypertension. The postoperative events are charted at irregular intervals, properly designated. The blood pressure charted 2 or 3 weeks following splanchnicectomy represents the values at the time the patient was discharged from the hospital following operation. When the blood pressure fluctuated appreciably, the extremes are plotted and the intervening space shaded. Although it is not a true representation, the values plotted for each occasion have been joined by a line in order to illustrate the trend of events.

In Figure 1 the changes in a patient (Case 1, W. L.) whose blood pressure after splanchnicectomy remained below $160 / 100 \mathrm{~mm}$. $\mathrm{Hg}$, are shown. The urea clearance was normal in this patient before operation, whereas the concentrating ability was greatly reduced. The lack of parallelism of these functions of the kidneys in this disease has been pointed out previously (6). This is an unusually wide variation, however. At the time of discharge from the hospital the urea clearance was below normal. A temporary decrease in urea clearance in the early postoperative period has been observed in a few other cases. Following splanchnicectomy, proteinuria promptly disappeared. Five months after operation the renal function had become entirely normal, and has remained so for more than two years. 


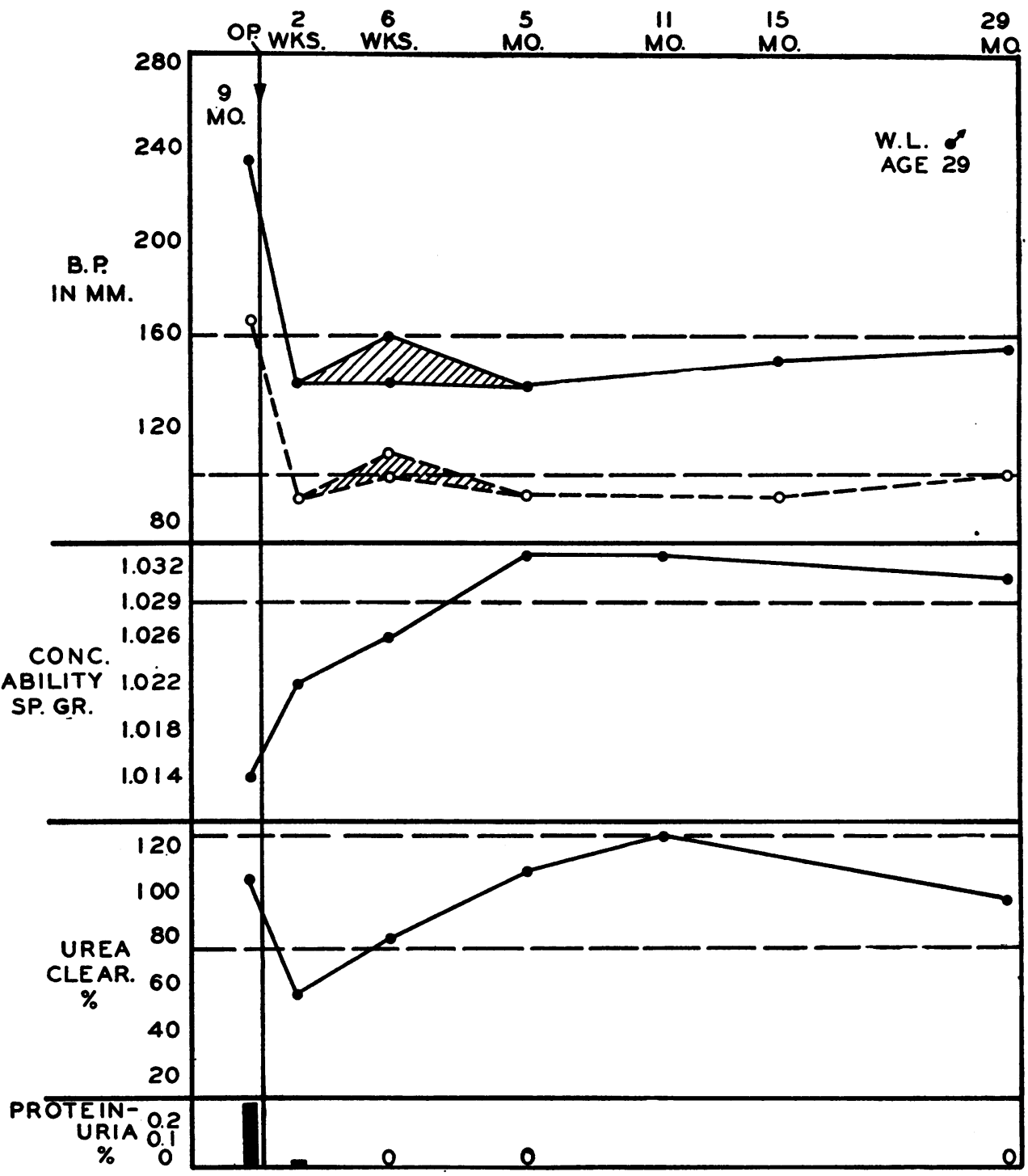

Fig. 1. Blood Pressure, Renal Function and Urinary Findings in Case 1 (W. L.) with MARKed ReLief of Hypertension.

Concentrating ability returned to normal following splanchnicectomy. 
TABLE II

The relation between renal function and blood pressure in 48 cases at the end of three months or longer after splanchnicectomy

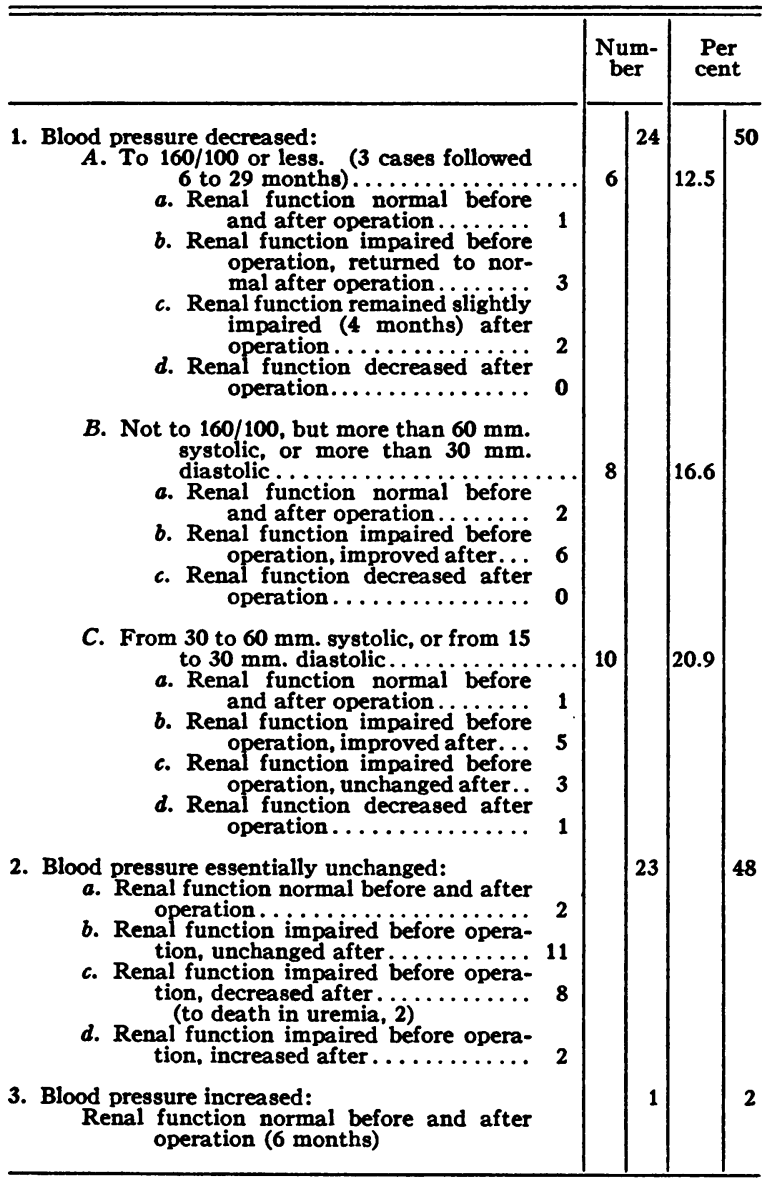

In Case 2, M. P. (Figure 2) there was a prompt return to normal of both the urea clearance and concentrating ability, and the disappearance of proteinuria, with relief of the hypertension.

In Case 4, V. E. (Figure 3) there was no impairment of renal function before operation, and the efficiency of the kidneys remained normal, or promptly returned to normal after a temporary decrease following splanchnicectomy, when the hypertension was relieved. This case illustrates that if urinary abnormalities exist, they may disappear or greatly decrease.

In many patients, blood pressure was markedly reduced, but not below 160/100 mm. Hg (Group $1, \mathrm{~B}$ of classification above). In such cases, if renal function had been normal, it remained so;

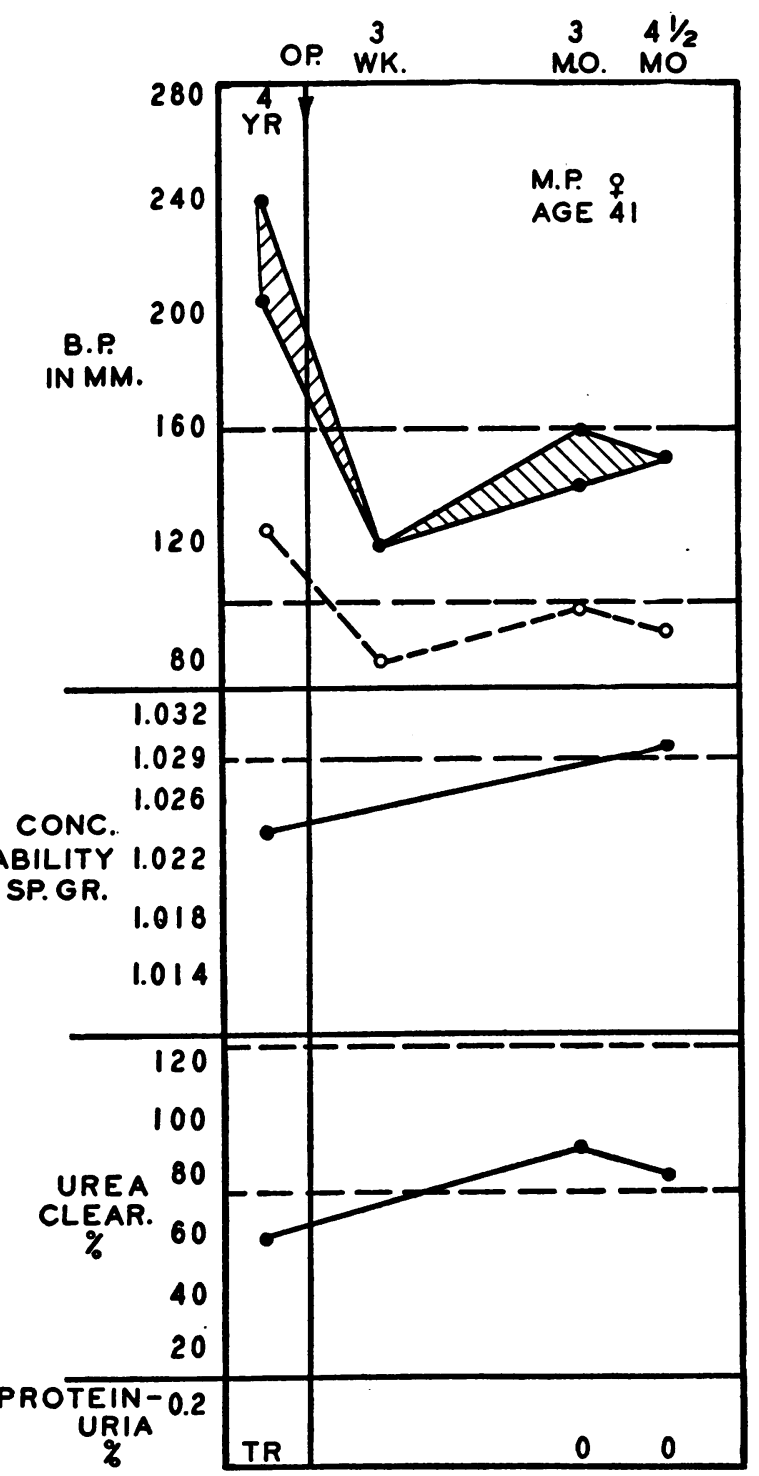

Fig. 2. Data in Case 2 (M. P.), Showing a Return to Normal of the Concentrating Ability and Urea Clearance with Relief of the Hypertension.

if it had been impaired, it improved and in some cases became entirely normal and the urinary abnormalities completely disappeared. Cases illustrating this effect are shown in Figures 4 and 5.

In some patients the blood pressure was decreased to a lesser degree (Group 1, C of classification above). In these persons, renal function usually improved if it had not been normal, or remained unchanged. In only one case (Case 24, L. B.) in this group was the concentrating ability found, fourteen months after operation, to be 


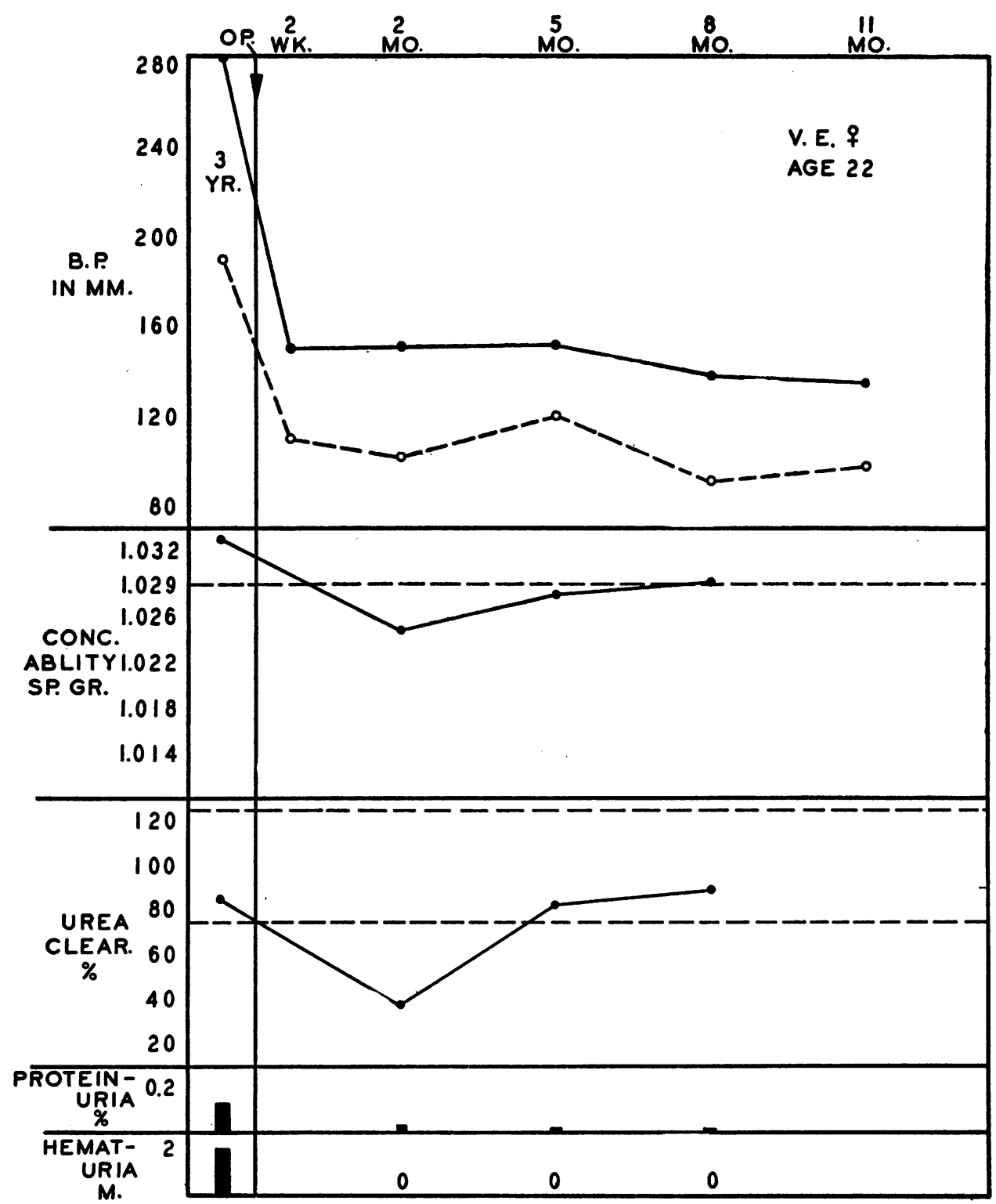

Fig. 3. Data in Case 4 (V. E.), Whose Renal Function Was Normal Before SPLANCHNICECTOMY. 


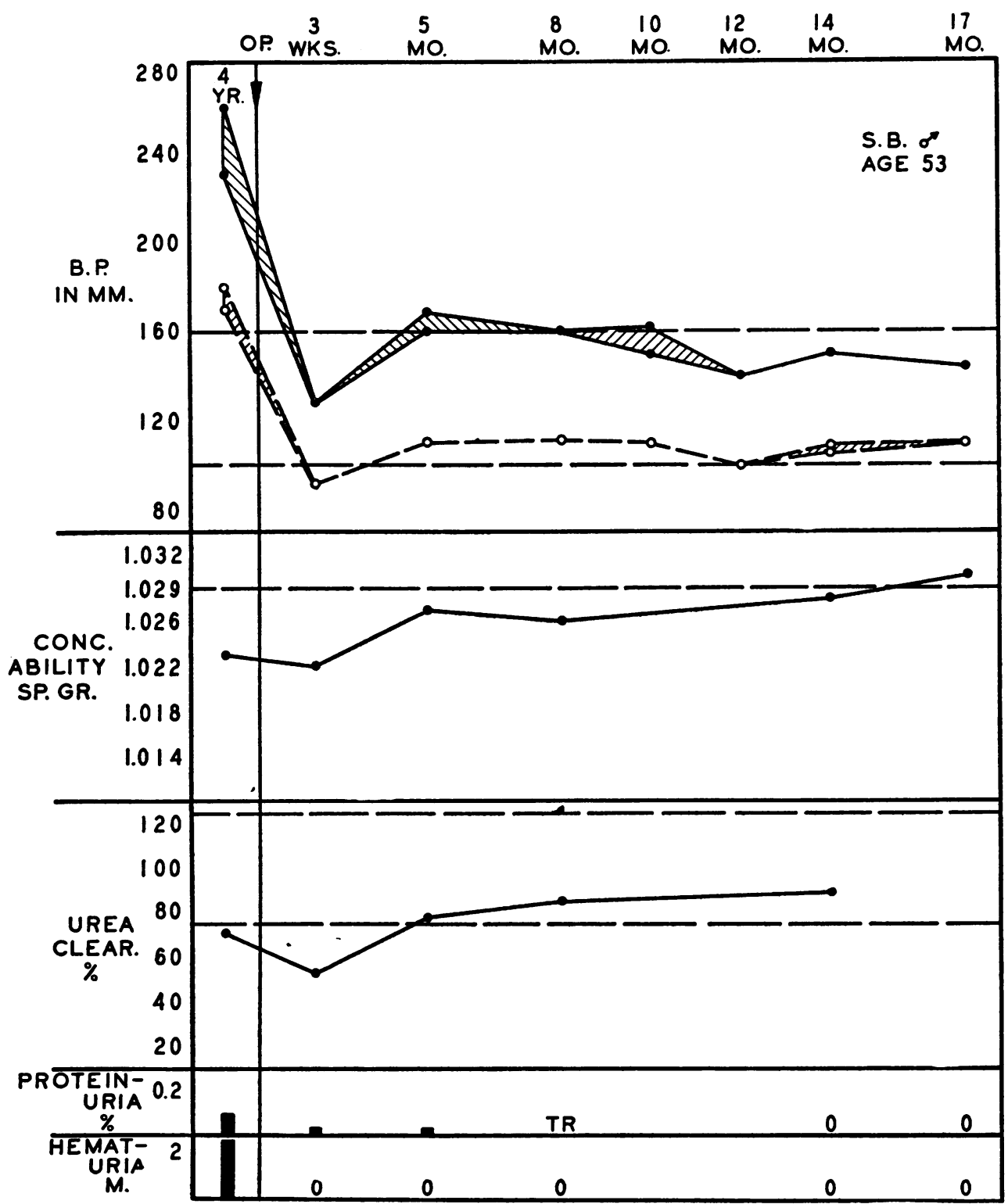

Fig. 4. Case 8 (S. B.), Showing Return to Normal Renal Function and Normal Urine with Lowering of Blood Pressure. 


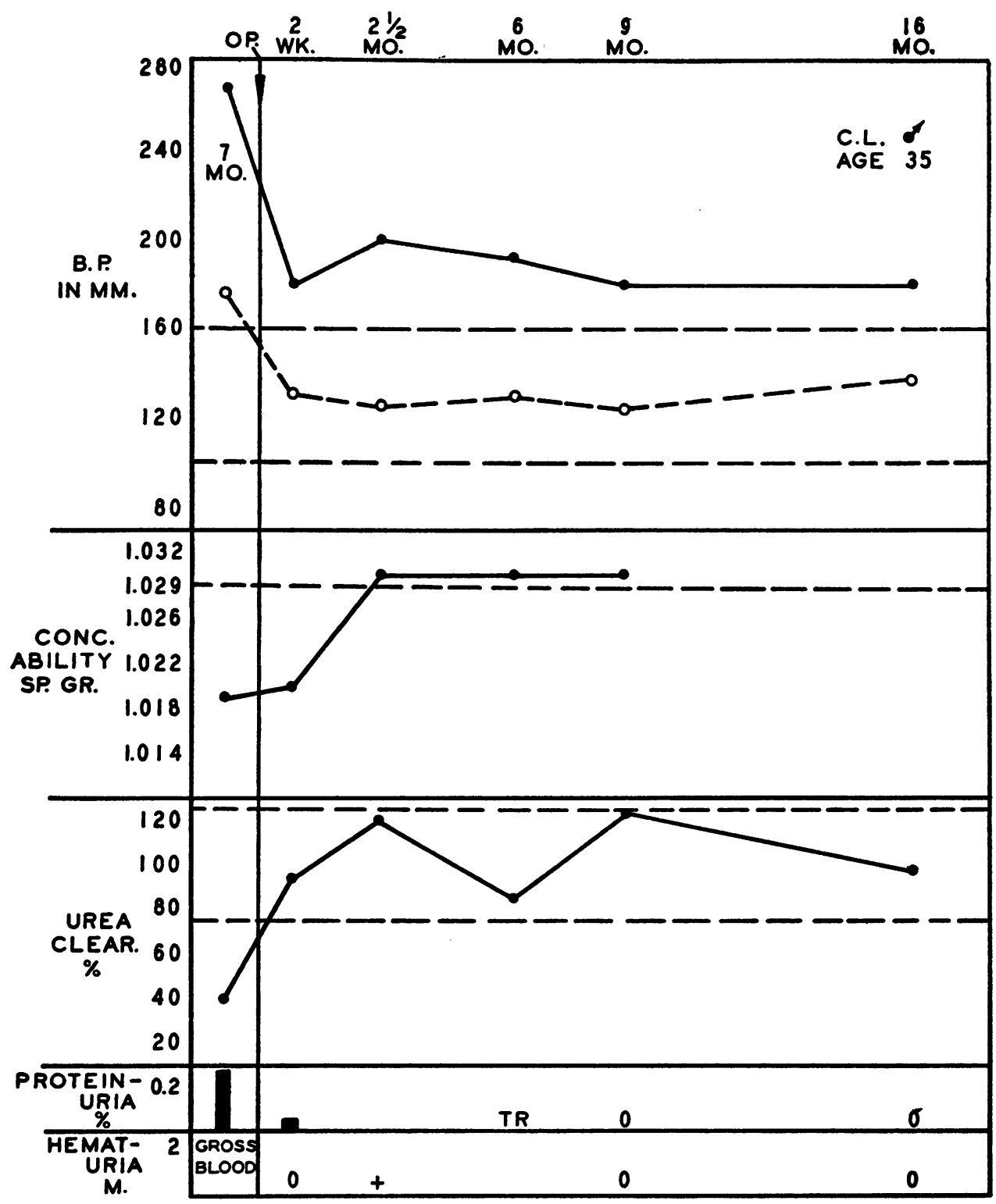

Fig. 5. Showing that Renal Activity and Urine Promptly Became Normal in Case 7 (C. L.) Whose Blood Pressure Was Reduced but not to the Low Levels Illustrated in Previous Charts. 


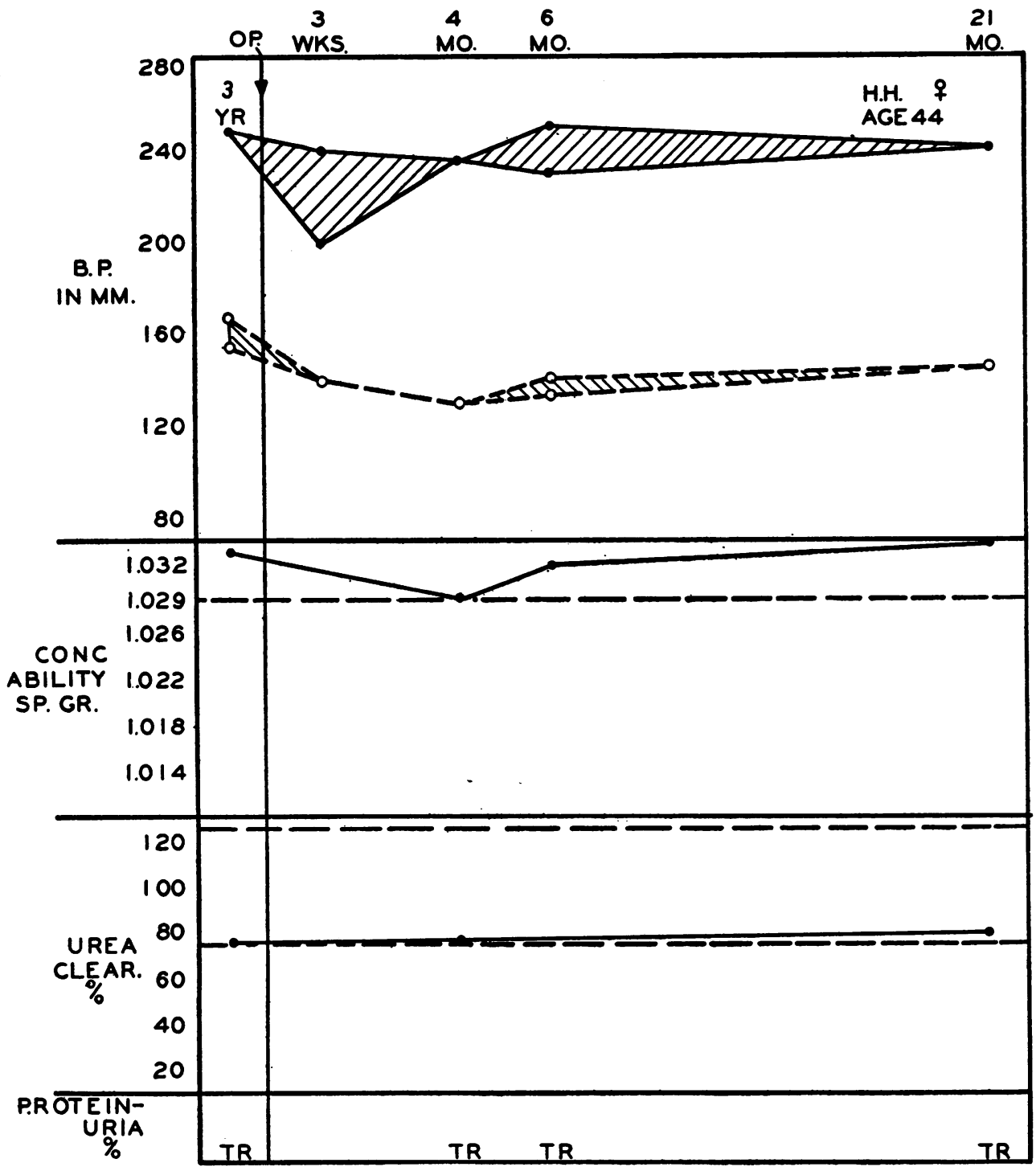

Fig. 6. Showing Renal Function Remaining Normal after Splanchnicectomy in Case 25 (H. H.) Who Had no Lasting Decrease in Blood Pressure. 


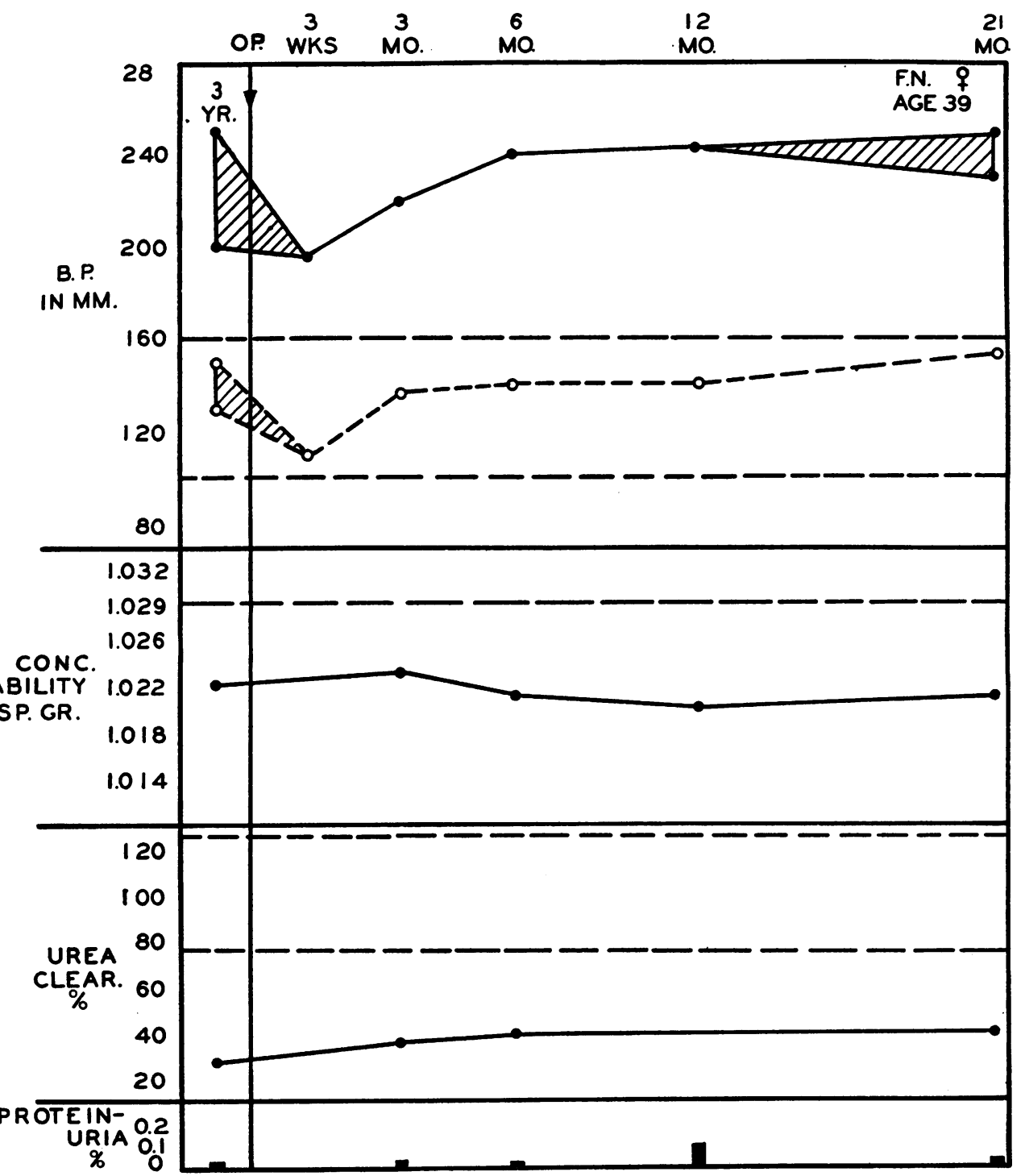

Fig. 7. Reduced Renal Function and Urine Remained Essentially Unchanged in Case 29 (F. N.) Who Had no Lasting Reduction in Blood Pressure. 


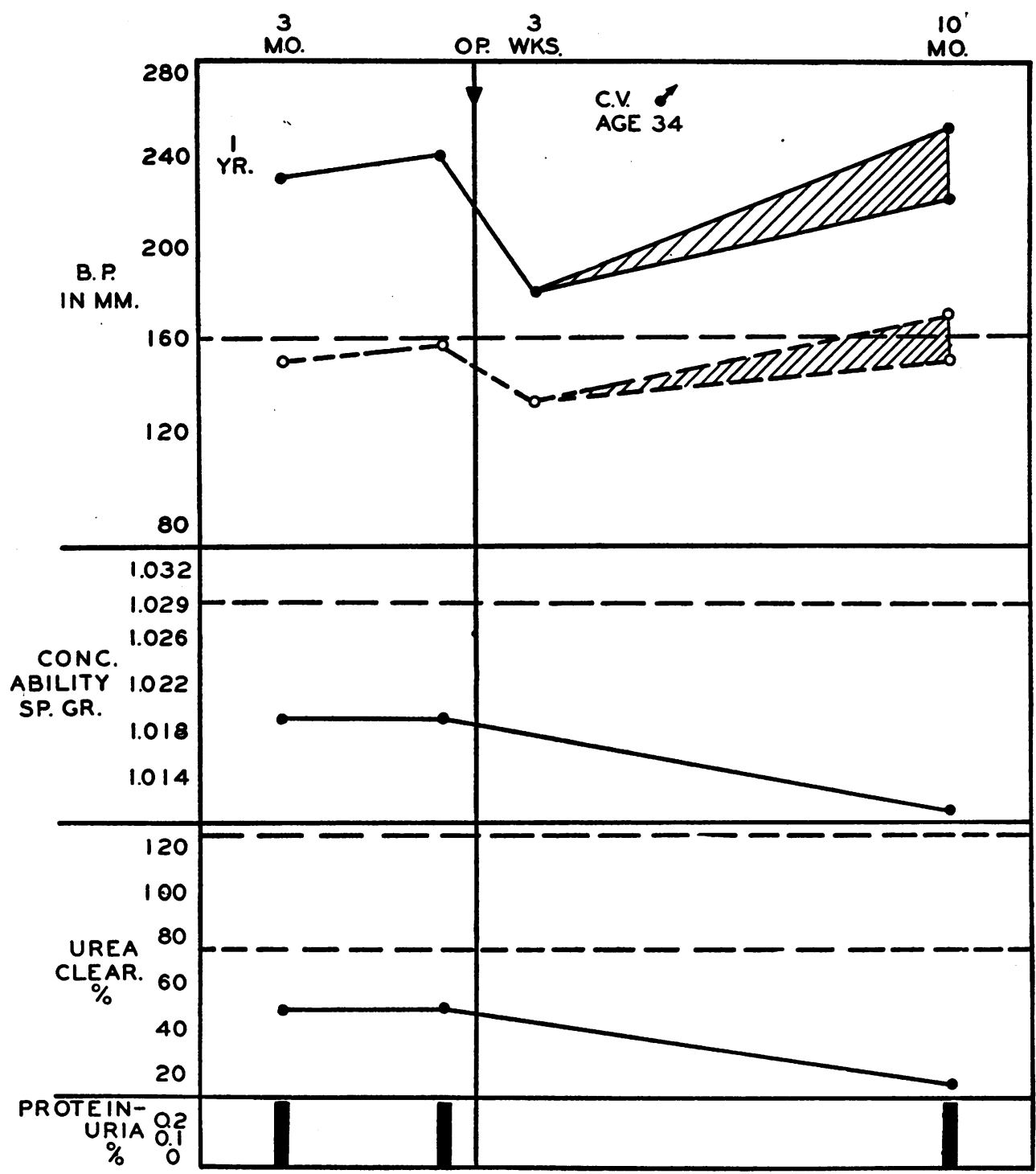

Fig. 8. Showing Decrease in Renal Function and Persistence of Proteinuria in Case 40 (C. V.) Whose Hypertension Was not Benefitted by Splanchnicectomy. 
slightly decreased. (The blood pressure at this time averaged $185 / 120 \mathrm{~mm}$. $\mathrm{Hg}$.)

In 50 per cent of the patients comprising this study, the blood pressure did not remain reduced following splanchnicectomy. In general, the renal function in this group of cases either remained unchanged, or became gradually worse, in a manner just as would be expected if splanchnicectomy had not been performed (Figures 6,7 and 8). In two such cases (Case 46, F. G., and Case 47, L. B.) renal function decreased progressively until death occurred in uremia. Two persons in this group (Case 27, H. S., and Case 28, T. A.) showed an improvement in renal function.

\section{DISCUSSION}

We wish to emphasize that this study is not meant to convey statistics regarding the effect of splanchnicectomy on blood pressure or other clinical results. Many more patients have been operated upon than we have been able to study in the manner here described. Symptomatic changes, ocular fundus and cardiac changes, mortality, etc., must of course be considered in a complete appraisal of results of this form of treatment. We have included data on blood pressure herein, and have grouped the patients according to changes in their blood pressure, only for convenience in discussion of the effect on the kidneys. Those persons whom we considered to have small decreases in blood pressure, and especially those whom we have been able to follow only three to six months, may be found after a longer period of postoperative study to have no lasting reduction in blood pressure. It should be noted, however, that in four patients (Cases 4, 7, 8 and 1) the blood pressure has remained at a remarkably low level in comparison with preoperative values, for 11,16 , 17 and 29 months, respectively, following splanchnicectomy.

From the data presented, it is evident that splanchnicectomy performed on patients with primary hypertension and normal kidney function, does not harm the kidneys, or interfere with their functional efficiency as measured by concentration and urea clearance test, whether or not significant decrease in blood pressure results. Page and Heuer (7) have likewise found that denervation of the kidneys which resulted from sectioning the anterior nerve roots from the 6th thoracic to the 2d lumbar segment in a patient with essential hypertension, in no way interfered with the renal function even though the blood pressure was reduced to normal. Page (8) has also shown that reduction in blood pressure induced by medication, or resulting from direct renal denervation of one kidney did not alter renal function as measured by urea clearance. All of these observations, therefore, show that in cases of primary hypertension, renal efficiency is not dependent on high blood pressure, as has been so commonly thought. Thus the " compensatory theory" of the cause of the elevated blood pressure in patients with primary hypertension is disproven.

We have repeatedly observed that when hypertension is greatly relieved by splanchnicectomy, renal function that has previously been impaired, improves, and may even return to normal. This improvement in kidney function has shown itself both by an increase in concentrating ability, and by an increase in urea clearance, in a number of cases. ${ }^{1}$ This indicates to us that the impairment of renal function is caused by vascular constriction, and that if constriction is relieved by splanchnicectomy, renal activity is benefitted.

In some cases we have observed, as have Page and Heuer (9), an improvement in proteinuria and hematuria, out of all proportion to changes in renal function.

That the results obtained by splanchnicectomy should differ so widely in different patients, from complete relief of hypertension, in some cases, to complete failure in others, is striking. We are at present unable to predict or account for these differences. Contrary to the findings of Page and Heuer (9), however, we find a definite association between changes in renal function and in blood pressure in most patients. Certainly, renal disease with marked impairment of function may accompany hypertensive vascular disease, and when present, disappears following relief of the hypertension by splanchnicectomy.

\footnotetext{
1 Page and Heuer (9) found an increase in concentrating ability in 2 of 5 patients with nephritis whose kidneys were denervated, but found no alteration in urea clearance.
} 


\section{SUM MARY}

Data regarding the renal status and blood pressure in 48 patients with hypertensive vascular disease treated by bilateral splanchnicectomy are presented. This surgical procedure has greatly relieved hypertension in some cases, has benefitted others to a lesser degree, and has not influenced the blood pressure in still others. In general, the changes in the kidneys were associated with changes in blood pressure. In those patients who had a significant and maintained decrease in blood pressure, urinary abnormalities decreased or disappeared, and the renal function, if it had been impaired, improved-in several cases it became entirely normal. In a few cases with less decrease in blood pressure, renal function remained unchanged following splanchnicectomy. When hypertension was lowered in patients having normal renal function, the efficiency of the kidneys remained normal. When hypertension was not favorably influenced, renal function remained unchanged, or gradually became worse as would be expected in unoperated cases.

These observations show that in cases of primary hypertension, satisfactory renal function is not dependent on the high blood pressure; that hypertension is not compensatory to measurable renal damage; that marked impairment of renal function may accompany hypertensive vascular disease, and that striking improvement of renal function follows relief of hypertension brought about by splanchnicectomy.

\section{BIBLIOGRAPHY}

1. Peet, M. M., Splanchnic section for hypertension. A preliminary report. Univ. Hosp. Bull., Ann Arbor, 1935, 1, 17.

2. Van Slyke, D. D., and Cope, C. L., Simplified colorimetric determination of blood urea clearance. Proc. Soc. Exper. Biol. and Med., 1932, 29, 1169.

3. Lashmet, F. H., and Newburgh, L. H., An improved concentration test of renal function. J. A. M. A., 1932, 99, 1396.

4. Lashmet, F. H., and Newburgh, L. H., An improved concentration test of renal function. II. Simple method for measuring proteinuria. J. A. M. A., 1933, 100, 1328.

5. Addis, T., A clinical classification of Bright's diseases. J. A. M. A., 1925, 85, 163.

6. Freyberg, R. H., The choice and interpretation of tests of renal efficiency. J. A. M. A., 1935, 105, 1575.

7. Page, I. H., and Heuer, G. J., The effect of renal denervation on the level of arterial blood pressure and renal function in essential hypertension. $J$. Clin. Invest., 1935, 14, 27.

8. Page, I. H., The effect on renal efficiency of lowering arterial blood pressure in cases of essential hypertension and nephritis. J. Clin. Invest., 1934, 13, 909.

9. Page, I. H., and Heuer, G. J., The effect of renal denervation on patients suffering from nephritis. J. Clin. Invest., 1935, 14, 443. 